\title{
Resting metabolic rate is increased in hypertensive patients with overweight or obesity: Potential mechanisms
}

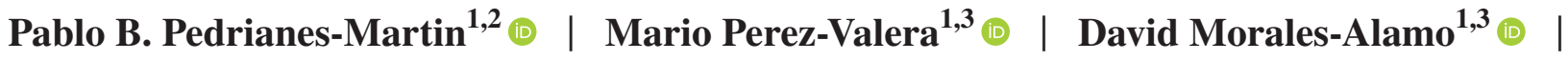

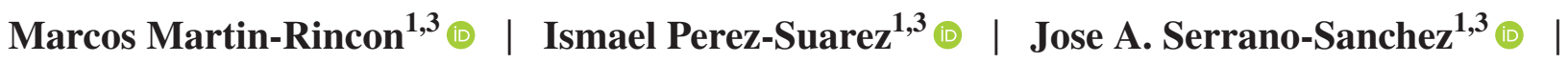 \\ Juan Jose Gonzalez-Henriquez $^{1,4}$ ｜ Victor Galvan-Alvarez ${ }^{1,3}$ ｜ Carmen Acosta ${ }^{1,2}$ | \\ David Curtelin $^{1} \odot$ | Pedro de Pablos-Velasco ${ }^{1,2} \mid$ Jose A. L. Calbet ${ }^{1,3,5} \odot$
}

${ }^{1}$ Research Institute of Biomedical and Health Sciences (IUIBS), Las Palmas de Gran Canaria, Spain

${ }^{2}$ Department of Endocrinology and Nutrition, Hospital Universitario de Gran Canaria Doctor Negrín, Las Palmas de Gran Canaria, Spain

${ }^{3}$ Department of Physical Education, University of Las Palmas de Gran Canaria, Las Palmas de Gran Canaria, Spain

${ }^{4}$ Department of Mathematics, University of Las Palmas de Gran Canaria, Las Palmas de Gran Canaria, Spain

${ }^{5}$ Department of Physical Performance, Norwegian School of Sport Sciences, Oslo, Norway

\section{Correspondence}

Jose A. L. Calbet, Department of Physical Education, University of Las Palmas de Gran Canaria, University Campus of Tafira, 35017 Las Palmas de Gran Canaria, Canary Islands, Spain. Email: lopezcalbet@gmail.com

\section{Funding information}

This study was financed by a grant from the Ministerio de Economía y Competitividad (PI14/01509 and FEDER), ULPGC (ULPAPD-08/01-4), and ACIISI (ProID2017010106)
The purpose of this investigation was to determine whether differences in body composition, pharmacological treatment, and physical activity explain the increased resting metabolic rate (RMR) and impaired insulin sensitivity in hypertension. Resting blood pressure, RMR (indirect calorimetry), body composition (dual-energy X-ray absorptiometry), physical activity (accelerometry), maximal oxygen uptake $\left(\mathrm{VO}_{2} \mathrm{max}\right)$ (ergospirometry), and insulin sensitivity (Matsuda index) were measured in 174 patients (88 men and 86 women; 20-68 years) with overweight or obesity. Hypertension (HTA) was present in 51 men (58\%) and 42 women (49\%) ( $p=.29)$. RMR was $6.9 \%$ higher in hypertensives than normotensives $\left(1777 \pm 386\right.$ and $1663 \pm 383 \mathrm{kcal} \mathrm{d}^{-1}$, $p=.044$ ). The double product (systolic blood pressure $\times$ heart rate) was $18 \%$ higher in hypertensive than normotensive patients $(p<.001)$. The observed differences in absolute RMR were non-significant after adjusting for total lean mass and total fat mass (estimated means: $1702 \mathrm{kcal} \mathrm{d}^{-1}$, CI: 1656-1750; and $1660 \mathrm{kcal} \mathrm{d}^{-1}$, CI: $1611-$ $1710 \mathrm{kcal} \mathrm{d}^{-1}$, for the hypertensive and normotensive groups, respectively, $p=.19$, HTA $\times$ sex interaction $p=.37$ ). Lean mass, the double product, and age were the variables with the higher predictive value of RMR in hypertensive patients. Insulin sensitivity was lower in hypertensive than in normotensive patients, but these differences disappeared after accounting for physical activity and $\mathrm{VO}_{2 \max }$. In summary, hypertension is associated with increased RMR and reduced insulin sensitivity. The increased RMR is explained by an elevated myocardial oxygen consumption due to an increased resting double product, combined with differences in body composition between hypertensive and normotensive subjects.

\section{K E Y W O R D S}

exercise, hypertension, obesity, overweight, resting energy expenditure 


\section{1 | INTRODUCTION}

Hypertension has been associated with an increased resting metabolic rate (RMR) $)^{1,2}$ and insulin resistance, ${ }^{1}$ potentially reflecting sympathetic ${ }^{3}$ and renin-angiotensin overactivation. ${ }^{4}$ Kunz et al. ${ }^{1}$ reported a 9\% higher RMR in obese hypertensive patients $(n=43)$ compared with obese normotensive controls $(n=27)$. However, this increased RMR was only observed in the hypertensive patients with obesity, while the non-obese patients with hypertension had a RMR similar to the normotensive controls. ${ }^{1}$ Obesity is associated with an increased fat-free mass (FFM) mostly due to a larger skeletal muscle mass. ${ }^{5}$ Since FFM is the main determinant of RMR, ${ }^{6}$ an increased FFM could account for the elevated RMR in obese hypertensives. ${ }^{1}$ Obesity is commonly associated with reduced physical activity and cardiorespiratory fitness, facilitating the development of insulin resistance and hypertension. ${ }^{7,8}$ Nevertheless, Kunz et al. ${ }^{1}$ did not check for the impact that differences in body composition, physical activity, and cardiorespiratory fitness could have had on their results and did not distinguish between treated and untreated hypertensive patients in their analyses. Likewise, an association between systolic blood pressure and RMR has been reported in a diverse sample including mostly normotensive young men and women of African origin, ${ }^{2}$ but no distinction was made in this study between obese and non-obese patients.

Moreover, no apparent impact of co-morbidities on the predicted RMR has been reported in cross-sectional studies. ${ }^{9}$ It remains unclear whether the association between hypertension and RMR is due to differences in body composition, reduced physical activity and cardiorespiratory fitness, or hypertension-related metabolic alterations. Since myocardial oxygen uptake $\left(\mathrm{MVO}_{2}\right)$ accounts for $11 \%$ of the RMR, ${ }^{10}$ any small increase in resting heart rate or systolic blood pressure could result in an increased energy expenditure, ${ }^{11}$ which could also contribute to explain an increased RMR in hypertensive patients. Furthermore, previous studies have not taken into consideration the potential confounding effects of pharmacological treatments on the association between hypertension and resting metabolic rate in obese patients, except for betablockade which has been reported to reduce RMR. ${ }^{1}$

Therefore, the primary aim of the present investigation was to determine whether the reported increased RMR in hypertensive patients with obesity is mostly accounted for by differences in body composition when compared with normotensives from the same population. Another aim was to find out whether an increased $\mathrm{MVO}_{2}$ could contribute to explain a higher RMR in hypertensive patients. We hypothesized that hypertensive overweight and obese patients would have an increased RMR, explained mainly by differences in FFM and fat mass (FM), physical activity, cardiorespiratory fitness, and $\mathrm{MVO}_{2}$, with no repercussion of pharmacological treatment in the observed relationships.

\section{$2 \mid$ METHODS}

\section{1 | Study design and participants}

The study population was composed of 174 patients (88 men and 86 women) with overweight or obesity from Gran Canaria, who agreed to participate in a registered clinical trial (ISRCTN11049554) to reduce their body weight with exercise and a low-calorie diet. The following inclusion criteria were applied: (A) sex: men and women; (B) age: 18 to 70 years old; (C) body mass index (BMI) $\geq 27 \mathrm{~kg} \mathrm{~m}^{-2}$; (D) no medical contraindications to exercise; (E) smoking less than 6 cigarettes per day; and $(\mathrm{F})$ in case of glucose intolerance or type 2 diabetes patients were only admitted if diagnosed within the last 5 years. Further details on inclusion/ exclusion criteria can be found in the ISRCTN registry. The starting cross-sectional data were recorded between June and October 2016. The study was performed conforming to the Declaration of Helsinki after ethical approval (Ref. 140187). Before given their written consent, the volunteers were informed about the purposes, risks, and benefits of the study. Ninety-three patients (51 men and 42 women) had hypertension, of whom 21 men and 23 women were under pharmacological treatment. Ten men (M) and 13 women (W) were smokers. Six men and 4 women had type 2 diabetes (6 of them controlled with metformin and 4 with diet and exercise). Three men and 8 women had asthma requiring only sporadic treatment with inhalers. Hypertensive patients received pharmacological treatment with diuretics $(5 \mathrm{M} / 12 \mathrm{~W}$ receiving angiotensin-converting enzyme inhibitors combined with diuretics and $3 \mathrm{M} / 9 \mathrm{~W}$ were treated with angiotensin receptor blockers combined with diuretics), beta-blockers $(3 \mathrm{M} / 4 \mathrm{~W})$, calcium channel blockers $(5 \mathrm{M} / 5 \mathrm{~W})$, angiotensinconverting enzyme inhibitors or angiotensin receptor blockers $(17 \mathrm{M} / 14 \mathrm{~W}$, of which $3 \mathrm{M} / 9 \mathrm{~W}$ also received diuretics). Statins $(8 \mathrm{M} / 7 \mathrm{~W})$ and fenofibrate $(1 \mathrm{M})$ were administered to 16 patients with hypercholesterolemia. Nine women were on oral contraceptives, and 20 women were postmenopausal.

\section{2 $\quad$ General procedures}

The general procedures have been previously published. ${ }^{12}$ Subjects were asked to refrain from exercise and avoid drinking beverages containing alcohol, caffeine, or taurine for the $48 \mathrm{~h}$ preceding blood sampling and testing. After a 12 -h overnight fast, participants reported to the laboratory between 07:00 and 09:30 a.m. Then, their body weight and height were measured to the nearest $0.1 \mathrm{~kg}$ and $0.1 \mathrm{~cm}$, respectively, while subjects wore light clothes and no shoes, using a balance scale (Seca) calibrated using certified calibration masses of class M1. This was followed by the assessment of their body composition by dual-energy X-ray absorptiometry 
(Lunar iDXA, General Electric), as previously reported. ${ }^{13}$ After that, their blood pressure was measured in triplicate (Omron M3 Intellisense HEM-7131-E) after a 5 min seatedperiod following the recommendations of the American Heart Association. ${ }^{14}$ After that, their RMR was determined by indirect calorimetry (see below). Resting heart rate (HR) was recorded continuously with a 12-lead PC-ECG extension of the metabolic carts. Upon termination of the RMR assessment, a 22-G cannula was inserted retrogradely in a heated hand vein, and basal blood samples were obtained. Then, they ingested $75 \mathrm{~g}$ of glucose dissolved in $300 \mathrm{ml}$ of water to assess their glucose tolerance (2-h test with sampling at 0, 30, 60,90 , and $120 \mathrm{~min}$ ). This was followed by an incremental cycle ergometer exercise test until exhaustion to determine their $\mathrm{VO}_{2 \max }$.

\subsection{Resting metabolic rate}

The RMR was determined by indirect calorimetry (Vmax N29; SensorMedics or Vyntus CPX;Jaeger-CareFusion) while the subjects rested quietly in the supine position for $30 \mathrm{~min}$ at an ambient temperature of $23-26^{\circ} \mathrm{C}$, as reported elsewhere. $^{12}$ The metabolic carts were calibrated immediately before each test according to the manufacturer's instructions, using certified high-grade calibration gases. The Vmax N29 SensorMedics has been validated for indirect calorimetry by the ethanol-burning test. ${ }^{15}$ In our laboratory, both metabolic carts slightly overestimated the stochiometric respiratory quotient of butane combustion, the Vmax N29 by $2.8 \%$ and the Vyntus by $1.5 \%$, with a coefficient of variation below $1 \%$ in both cases. ${ }^{16}$ All Vyntus CPX data were converted into Vmax N29 data, using data obtained with both analyzers for cross-calibration. Oxygen uptake $\left(\mathrm{VO}_{2}\right)$ and carbon dioxide production $\left(\mathrm{VCO}_{2}\right)$ were recorded breath by breath for 20 min after an initial 10-min habituation period using a face mask. Breath-by-breath data were averaged every $20 \mathrm{~s}$. All 20-s averages with $\mathrm{VO}_{2}$ values deviating from the mean more than two SD were discarded. Then, the mean $\mathrm{VO}_{2}$ and $\mathrm{VCO}_{2}$ values recorded during a 10-min period with steady $\mathrm{VO}_{2}$ were averaged to calculate the daily resting energy expenditure. ${ }^{17}$

\subsection{Maximal oxygen uptake}

All volunteers performed an incremental exercise test until volitional exhaustion with verification ${ }^{18}$ on a cycle ergometer (Corival, Lode). The exercise test started at $20 \mathrm{~W}$ for $3 \mathrm{~min}$, followed by $10 \mathrm{~W}$ increments every $3 \mathrm{~min}$ until the respiratory exchange ratio (RER) was $\geq 1.00$. After that, the cycle ergometer was unloaded, and subjects were allowed to recover for $2 \mathrm{~min}$ while remaining seated on the cycle ergometer pedaling at a low cadence (40-50 rpms). After that, the exercise intensity was raised to the same intensity at which the RER of 1.00 was attained, and maintained for one minute. Then, the intensity was increased by $10 \mathrm{~W}$ (women) or $15 \mathrm{~W}$ (men) every minute until exhaustion. Upon exhaustion, the ergometer was unloaded while the subjects recovered pedaling at a low cadence $(40-50 \mathrm{rpm})$. At the $3 \mathrm{~min}$ of the recovery phase, a verification phase was initiated by bringing the ergometer to the intensity at which exhaustion had occurred in the previous phase $+5 \mathrm{~W}$ for $1 \mathrm{~min}$, continued by $5 / 4 \mathrm{~W}$ increments in men/women, respectively, every $20 \mathrm{~s}$ until exhaustion. During the exercise test, subjects were advised to maintain the pedaling cadence at $70 \pm 5 \mathrm{rpm}$. Ergospirometric variables were averaged every $20 \mathrm{~s}$, and the highest 20-s averaged $\mathrm{VO}_{2}$ values recorded during the entire test were taken as the $\mathrm{VO}_{2 \max } \cdot{ }^{19}$ The $\mathrm{VO}_{2 \max }$ results are reported in absolute values $\left(\mathrm{ml} \mathrm{min}^{-1}\right)$ and normalized to body mass ( $\mathrm{ml} \mathrm{kg}^{-1} \mathrm{~min}^{-1}$ and normalized for RMR, ie, as METS).

\section{5 | Physical activity}

Physical activity was registered during four consecutive days, including two weekend days, using a Garmin Vivofit activity tracker wore on the non-dominant wrist (micro-electromechanical triaxial accelerometer) (Garmin International Inc).

\subsection{Statistical analysis}

Variables were checked for Gaussian distribution using the Kolmogorov-Smirnov test, and when appropriate, data were transformed logarithmically before further analysis. A chi-square test was used to determine whether there was a difference in the prevalence of hypertension between sexes, as well as if there was a difference in the percentage of pharmacologically treated hypertensives by sex. General characteristics of men and women were compared using an unpaired t test (reported in Table 1). Groups were categorized according to hypertension in three categories (treated, untreated, normotensive). Hypertensive groups were checked for differences in RMR using ANOVA (reported in Tables 2 and 3). This analysis was followed by ANCOVA, with FFM and FM as covariates and hypertension (treated, untreated, and absent) and sex (male and female) as between-subject factors. Additional ANCOVA analyses were performed considering age and physical activity or $\mathrm{VO}_{2 \max }$ (as an index of cardiorespiratory fitness) as covariates. The same approach was also carried after collapsing the untreated and treated hypertensive patients into a single group, to check for differences between normotensive and hypertensive patients, regardless of 
TABLE 1 Characteristics of the study population

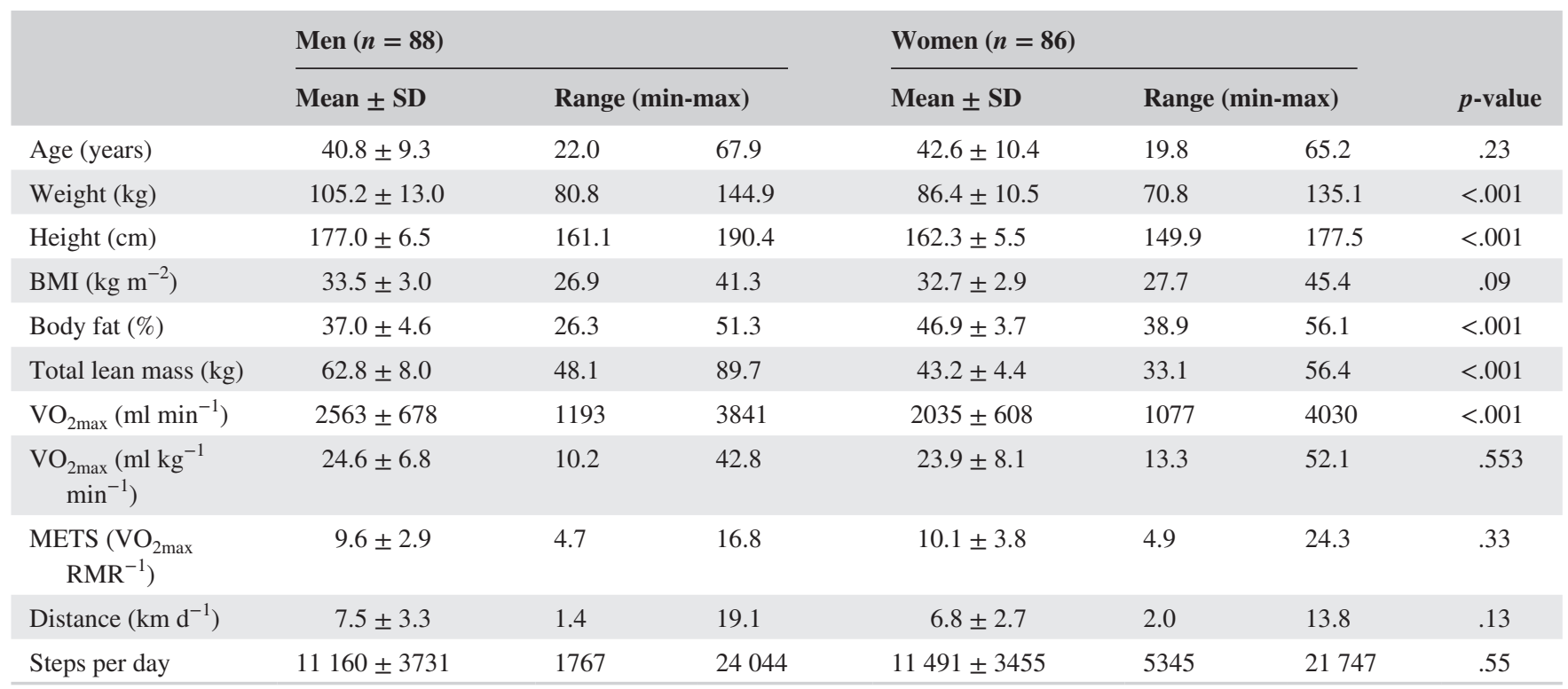

Note: Analysis based on unpaired $t$ test.

Abbreviations: BMI, body mass index; Distance, distance walked or run every day; METS, metabolic equivalents achieved during the incremental exercise to exhaustion; RMR, resting metabolic rate; Steps $\mathrm{d}^{-1}$, number of steps performed every day.

pharmacological treatment. Multiple stepwise linear regression was used to determine which variable had predictive value for RMR in patients with and without hypertension. Values are reported as the mean \pm standard deviation (SD) unless otherwise stated. Statistical significance was set at $p<.05$. All statistical analyses were performed using IBM SPSS Statistics v.21 for Mac (SPSS Inc).

\section{3 | RESULTS}

The descriptive characteristics of the study population are reported in Tables 1 and 2 . The prevalence of hypertension was similar in both sexes $(p=.29)$, and non-significant differences were observed in the proportion of hypertensive men and women under pharmacological treatment $(p=.22)$. Men and women had similar age and BMI, while women had a greater percentage of body fat than men. Compared to the normotensive volunteers, the hypertensive had a $15 \%$ and $13 \%$ higher diastolic and systolic arterial blood pressure values, respectively $(p<.001)$. Men had a slightly better aerobic fitness than women when the $\mathrm{VO}_{2 \max }$ was normalized to total lean body mass. Ninety-three subjects had hypertension, of which 44 were under pharmacological treatment, while the rest were not taking drugs. The 81 additional participants had normal resting blood pressure (Table 3). Despite the pharmacological treatment, the resting BP values were elevated in the treated group, so the BPs of both hypertensive groups were similar. Resting heart rate was slightly higher in the hypertensive compared to the non-hypertensive subjects $\left(66.0 \pm 8.9\right.$ and $63.4 \pm 7.8$ beats $\left.\min ^{-1}, p=.047\right)$, as was the double product (systolic blood pressure $\times$ heart rate) (Table 3).

Insulin sensitivity, as reflected by the resting fasting glucose concentration, the disposition index and the Matsuda index were slightly impaired in the hypertensive patients under treatment, while the insulinogenic index was similar in the three groups (Table 3). The differences in insulin sensitivity disappeared after accounting for lean body mass, fat mass, age and physical activity (distance walked every day) (for resting fasting glucose concentration $p=.17$, HTA $\times$ sex interaction $p=.81$; disposition index $p=.28$, HTA $\times$ sex interaction $p=.60$; Matsuda index $p=.17$, HTA $\times$ sex interaction $p=.49$ ). Similar results were obtained after accounting for lean body mass, fat mass and cardiorespiratory fitness $\left(\mathrm{VO}_{2 \max }\right.$ in $\mathrm{ml} \mathrm{kg} \mathrm{LM}{ }^{-1} \mathrm{~min}^{-1}$ ) (for resting fasting glucose concentration $p=.08$, HTA $\times$ sex interaction $p=.62$; disposition index $p=.14$, HTA $\times$ sex interaction $p=.75$; and Matsuda index $p=.07$; HTA $\times$ sex interaction $p=.30$ ).

Since no significant differences were observed in RMR values between treated and untreated hypertensive patients, the two hypertensive groups were collapsed into a single hypertensive group and compared with the normotensive patients. In absolute values, the RMR was 6.9\% higher in the hypertensive than normotensive group $(1777 \pm 86$ and $1663 \pm 383 \mathrm{kcal}$ $\left.\mathrm{d}^{-1}, p=.044\right)$. The observed differences in absolute RMR were non-significant after adjusting for total lean mass and total fat mass (estimated means: $1702 \mathrm{kcal} \mathrm{d}^{-1}$, CI: 16561750 and $1660 \mathrm{kcal} \mathrm{d}^{-1}, \mathrm{CI}$ : 1611-1710, for the hypertensive and normotensive groups, respectively, $p=.19$, HTA $\times$ sex interaction $p=.37$ ). Adding the age (ANCOVA HTA effect $p=.05$ ), or distance walked daily (ANCOVA HTA effect 
TA B LE 2 Body composition, fitness, and physical activity

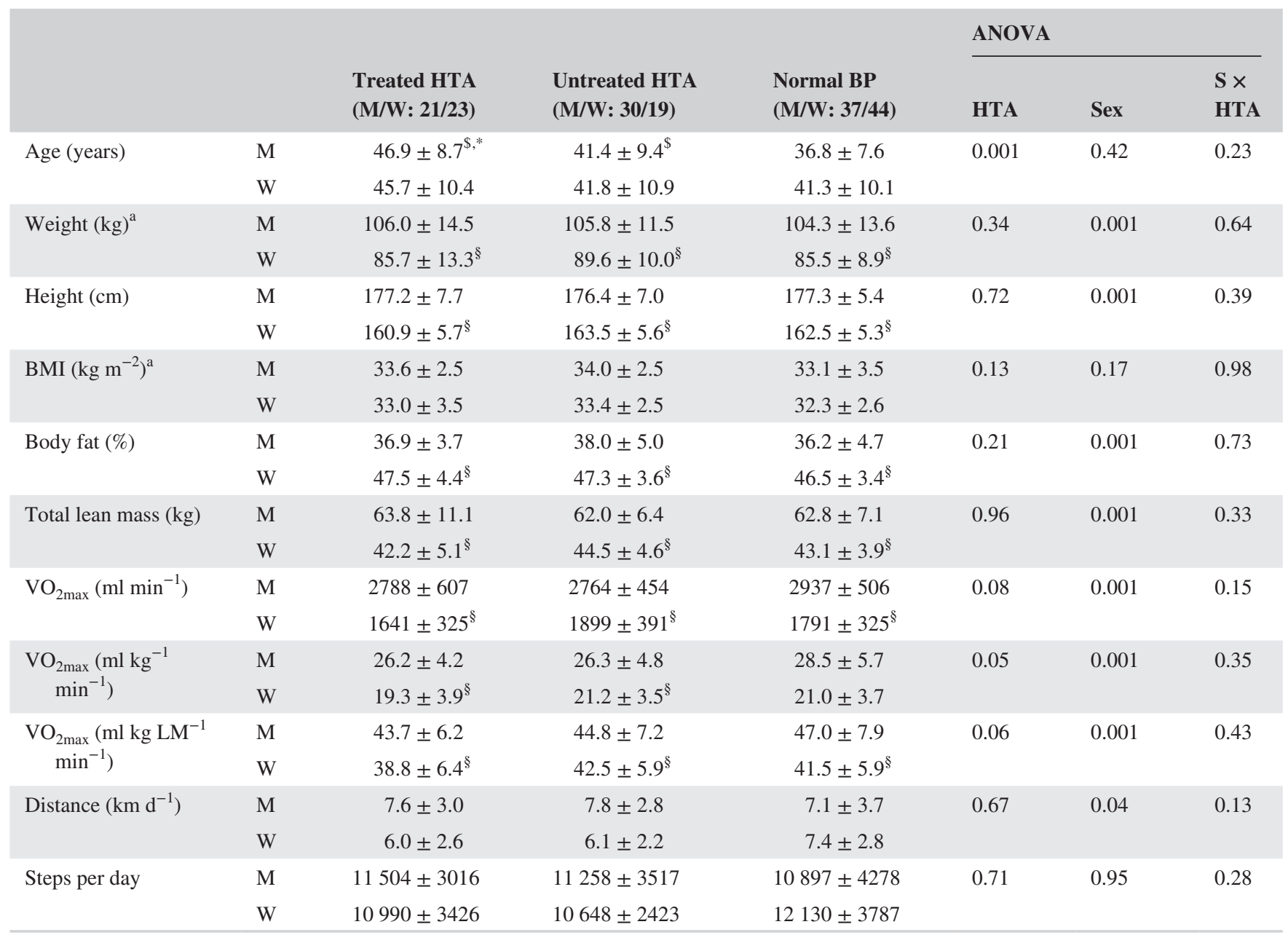

Abbreviations: BMI, body mass index; HTA, hypertension; LM, whole-body lean mass; M, men; VO ${ }_{2 \text { max }}$, maximal oxygen uptake; W, women.

${ }^{a}$ Statistical analysis after logarithmic transformation. Based on ANOVA (no covariates introduced), HTA: main effect for blood pressure group; Sex: main effect for differences between men and women; $\mathrm{S} \times$ HTA: sex by blood pressure interaction.

$\$ p<.05$ compared to the normal pressure group.

*Compared to the untreated hypertensives.

$\S_{p}<.05$ women compared to men.

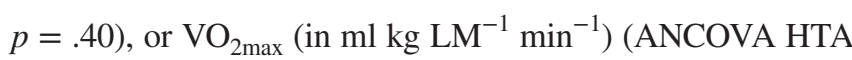
effect $p=.12$ ) or age and distance walked daily (ANCOVA HTA effect $p=.16$ ), or age and $\mathrm{VO}_{2 \max }$ (ANCOVA HTA effect $p=.07$ ), did not change significantly the results obtained. Moreover, no significant HTA $\times$ sex interactions were observed for adjusted RMRs. The same type of analysis restricted to subjects with BMI $>30 \mathrm{~kg} \mathrm{~m}^{-2}$ yielded similar results, that is, non-significant differences in RMR between normotensive and hypertensive obese subjects regardless of treatment status.

Multiple regression analysis indicates that the variable with the highest predictive value of RMR in hypertensive and normotensive patients was the whole-body lean mass (Table 4). Age was a negative predictor for RMR in both groups (Table 4). In hypertensive patients, the double product also had predictive value for the RMR. When all patients were analyzed combined, the whole-body fat mass was also a contributing factor to RMR (Table 5).

\section{4 | DISCUSSION}

This study shows that overweight and obese patients with hypertension have a 7\% higher RMR in absolute values compared to overweight and obese patients of similar characteristics without hypertension. However, no significant differences in RMR were observed between the two groups after accounting for interindividual differences in lean mass, fat mass, age, physical activity, and cardiorespiratory fitness. We have also shown that hypertensive patients with overweight or obesity have an increased double product at rest, which explains the observed increased RMR partly. This may reflect an increased sympathetic overactivation ${ }^{3}$ not sufficiently counteracted by the medical treatment. Overall, these findings indicate that hypertension per se has a minimal impact on RMR, which is primarily explained by the increased myocardial $\mathrm{VO}_{2}$ at rest, due to the increased work of 
T A B L E 3 Blood pressure, metabolic variables, and resting energy expenditure

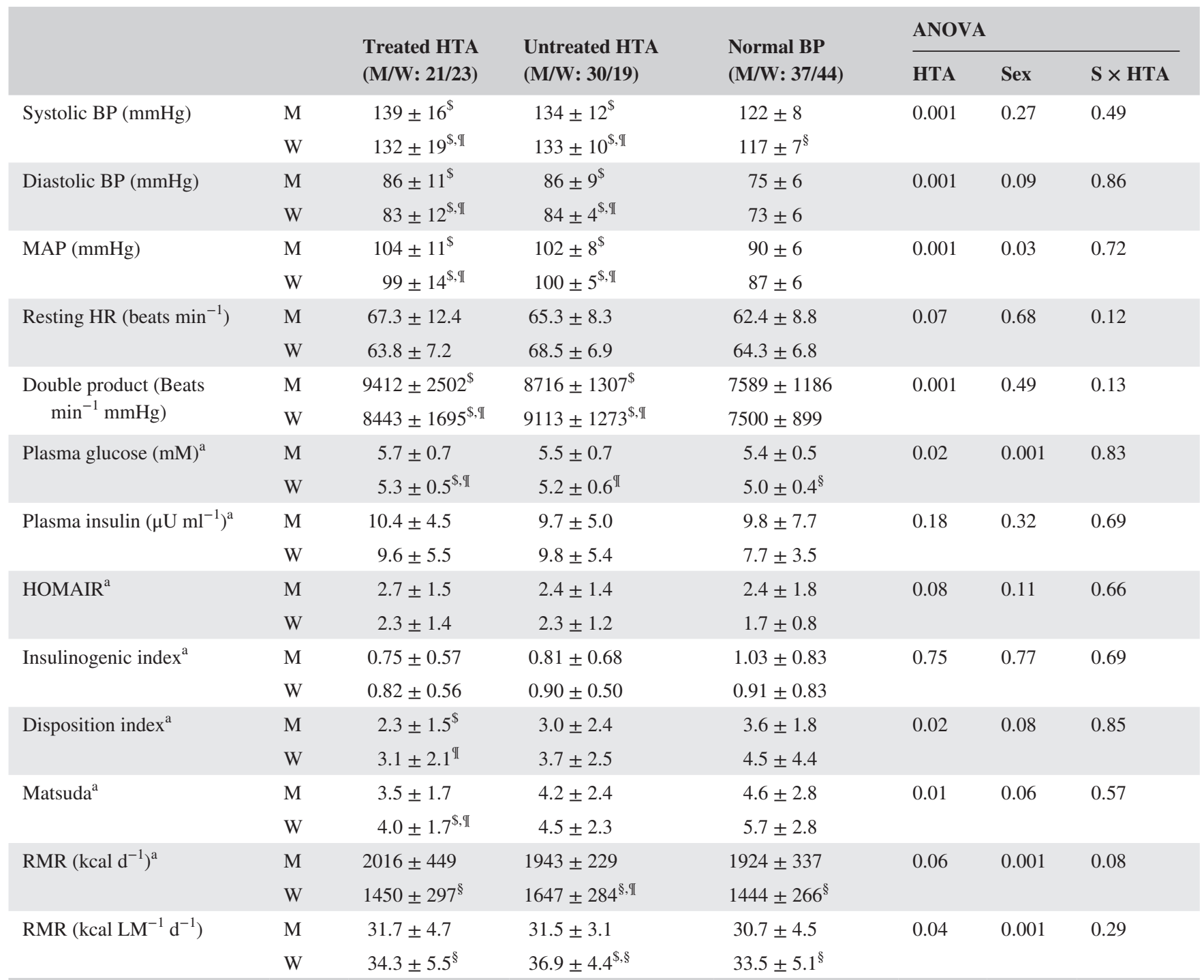

Abbreviations: BP, blood pressure; HTA, hypertension; LM, whole-body lean mass; M, men; MAP, mean arterial pressure; RMR, resting metabolic rate; W, women. ${ }^{a}$ Statistical analysis after logarithmic transformation. Based on ANOVA (no covariates introduced), HTA: main effect for blood pressure groups; Sex: main effect for differences between men and women; $\mathrm{S} \times$ HTA: sex by blood pressure interaction.

${ }^{\$} p<.05$ compared to the normal pressure group.

$\S_{p}<.05$ women compared to men.

${ }^{\mathbb{I}} p<.05$ both genders combined compared to the normal pressure group.

the heart caused by the elevated resting heart rate and systolic blood pressure.

\subsection{Hypertension per se has a small repercussion on resting metabolic rate}

The prevalence of hypertension in our study population (53\%) was slightly higher than reported for the Spanish adult population (43\%). ${ }^{20}$ Assuming a type 2 error, a $2 \%-3 \%$ higher RMR after accounting for lean and fat mass, could be ascribed to hypertension. This possibility is supported by the fact that the double product was a predictive variable for the RMR only in the hypertensive population. Our findings are in agreement with the pioneering study of Kunz et al. ${ }^{1}$ who observed a 9\% higher RMR in obese hypertensive patients $(n=43)$ compared with obese normotensive controls $(n=27)$. However, Kunz et al. ${ }^{1}$ did not check for the impact that differences in lean and fat mass could have had on their results and did not distinguish between treated and untreated hypertensives.

In the present investigation, we have observed that the double product measured at rest has predictive value for RMR in hypertensive patients. This is expected since the myocardial $\mathrm{O}_{2}$ uptake $\left(\mathrm{MVO}_{2}\right)$ is proportional to the double product $^{11}$ and accounts for $11 \%$ of RMR in healthy humans. ${ }^{10}$ 
T A B L E 4 Stepwise multiple regression models predicting resting energy expenditure in overweight or obese patients with or without hypertension

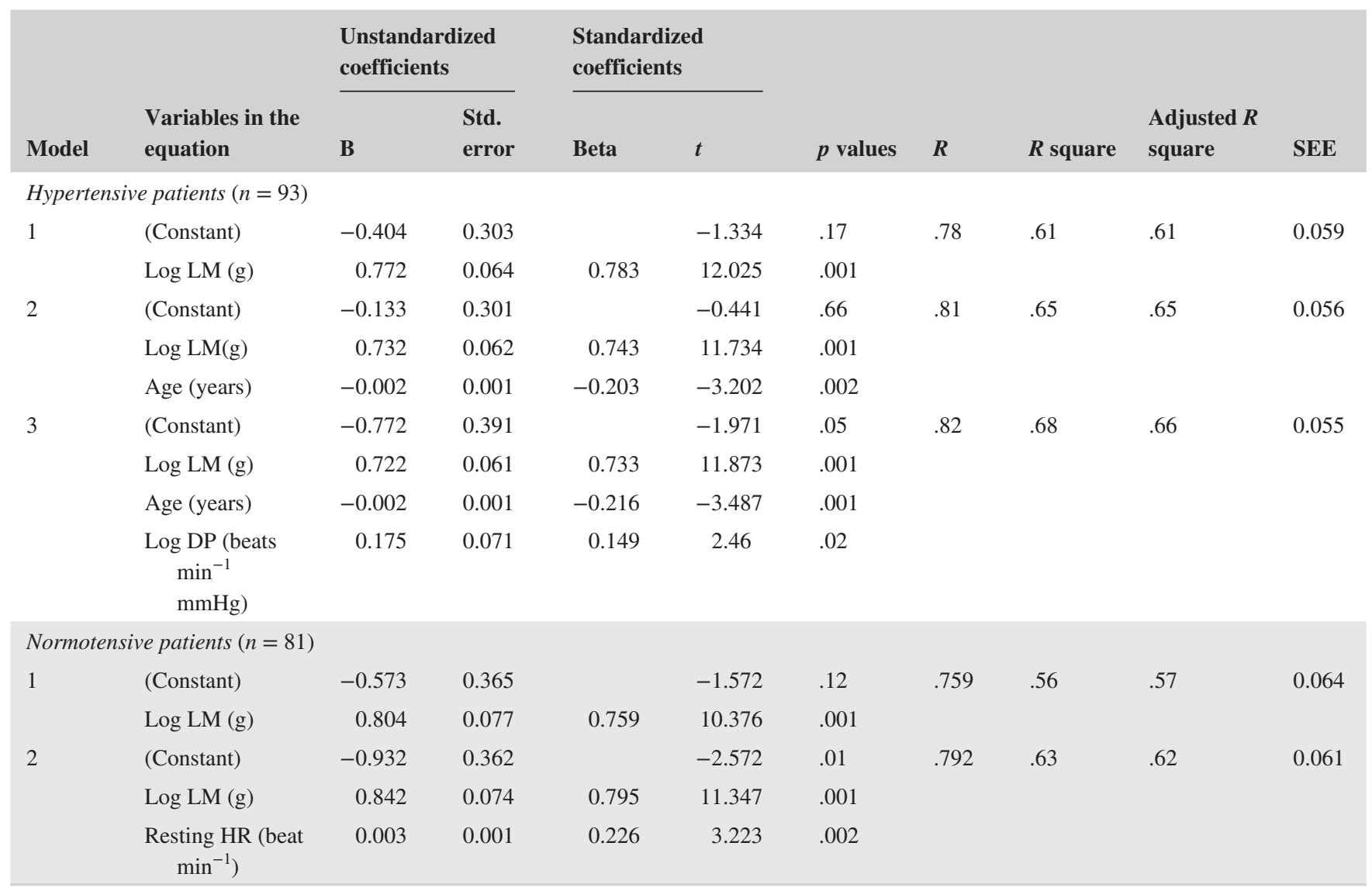

Note: Dependent variable: Logarithm of RMR $\left(\mathrm{kcal} \mathrm{d}^{-1}\right)$; for model 3, the following variables were excluded from the equation: Log FM, Resting HR, and Log BPsys. Dependent variable, Logarithm of RMR $\left(\mathrm{kcal} \mathrm{d}^{-1}\right)$; For model 2, the following variables were excluded from the equation: Log FM, Age, Log BPsys, and Log DP. Abbreviations: BPsys, Systolic blood pressure; DP, double product; FM, whole-body fat mass; LM, whole-body lean mass; Log, logarithm; SEE, standard error of estimate.

By applying the equation of Hoeft et al., ${ }^{11}$ we have estimated that the hypertensive patients had $1.3 \mathrm{ml} \mathrm{min}^{-1} 100 \mathrm{~g}^{-1}$ higher $\mathrm{MVO}_{2}$ than the normotensive, which is equivalent to a $15 \%$ higher $\mathrm{MVO}_{2}$ at rest. The latter would add $21 \mathrm{kcal} \mathrm{d}^{-1}$ to the RMR (equal to a 1.2\% higher RMR). These calculations assume minimal differences in heart mass between groups, after accounting for body size. ${ }^{21}$

Although not measured here, sympathetic overactivity has also been suggested as a contributing factor to enhancing RMR in hypertension and obesity, ${ }^{22,23}$ as supported by epidemiological data. ${ }^{24,25}$ However, after accounting for the direct effect of $\mathrm{MVO}_{2}$ on RMR, other factors not directly associated with lean mass and fat mass could at best explain about $50 \%$ of the difference in RMR between hypertensive and normotensive.

\subsection{Fat mass explains part of the differences in RMR due to hypertension}

Both the analysis of covariance and the multiple regression analysis, indicate that fat mass contributes significantly to the
RMR, explaining part of the $7 \%$ greater RMR observed in the patients with hypertension. An increased fat mass may raise the RMR directly due to the $\mathrm{VO}_{2}$ of the adipocytes, which becomes more physiologically relevant the higher the fat mass. ${ }^{10,26}$ Indirectly, the fat mass may rise the RMR through the central effects of hyperleptinemia, ${ }^{27,28}$ which stimulates sympathetic activity ${ }^{29}$ and induces the activation of hypothalamic-pituitary-thyroid axis increasing the circulating levels of thyroid hormones. ${ }^{10,30-32}$

\section{3 | Hypertension is associated with insulin resistance}

In agreement with the seminal study of Kunz et al., ${ }^{1}$ the hypertensive patients studied here had lower insulin sensitivity than the normotensive; nevertheless, these differences were attenuated and even disappeared in the case of the Matsuda index, after accounting for physical activity or cardiorespiratory fitness. However, in contrast with Kunz et al., ${ }^{1}$ non-significant differences were noted here between 
T A B L E 5 Stepwise multiple regression models predicting resting energy expenditure in overweight or obese patients (all patients analyzed conjointly, $n=174)$

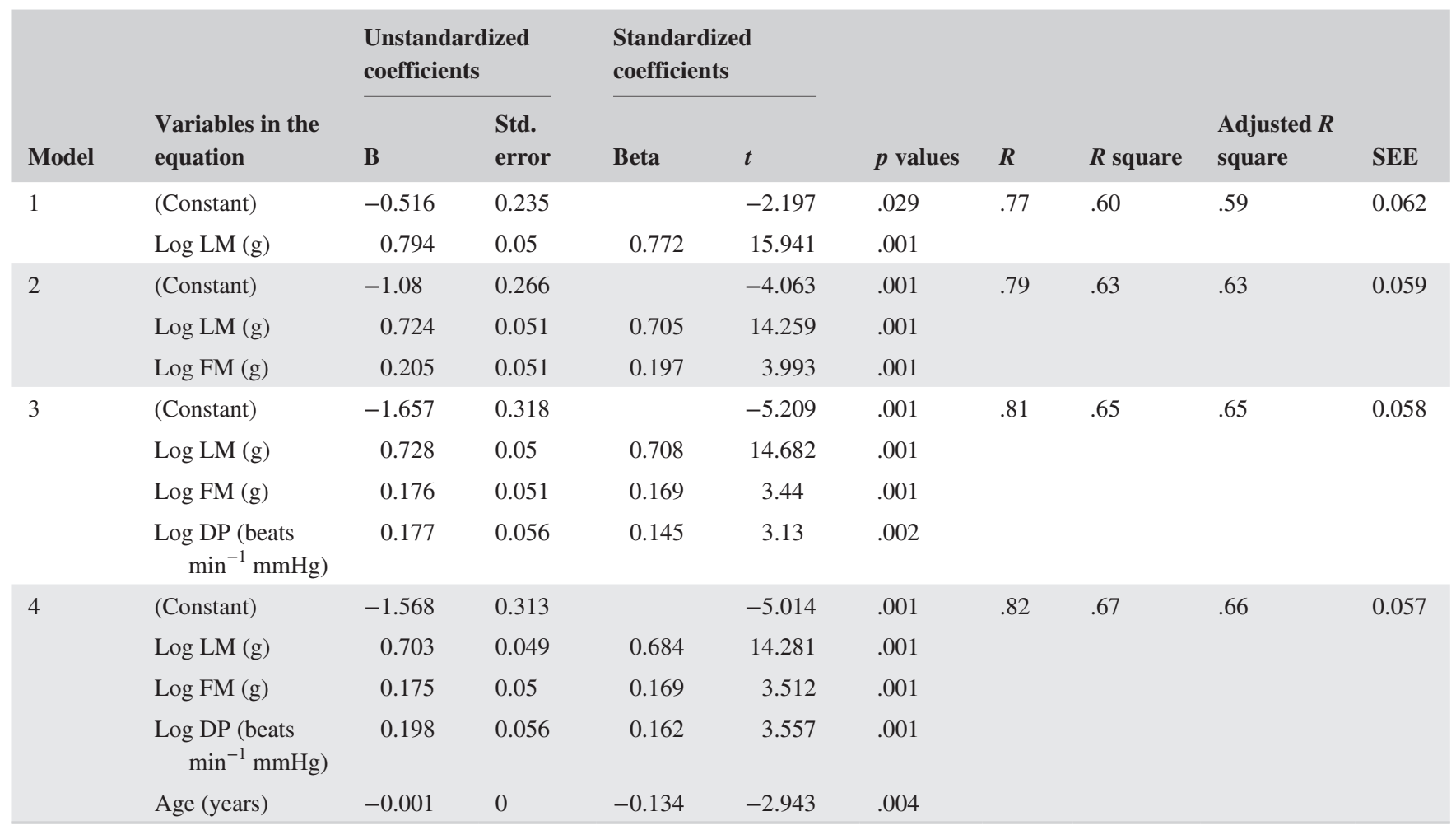

Note: Dependent variable, Logarithm of RMR ( $\mathrm{kcal} \mathrm{d}^{-1}$ ); For model 4, the following variables were excluded from the equation: Resting HR and Log BPsys. Abbreviations: BPsys, Systolic blood pressure; DP, double product; FM, whole-body fat mass; LM, whole body lean mass; Log, logarithm; SEE, standard error of estimate.

hypertensives and normotensives in resting insulin concentrations, after accounting for lean and fat mass. Given the impact that physical activity has on insulin sensitivity, ${ }^{33,34}$ our results emphasize the importance of increasing physical activity in hypertensive patients to attenuate the effect of hypertension on insulin sensitivity.

Hypertension may reduce insulin sensitivity by multiple mechanisms, including impairment of endothelial function and insulin transport across the endothelium, ${ }^{35,36}$ impairment of organ blood flow and microvascular blood flow regulation in response to nutrients, ${ }^{37,38}$ reduced nitric oxide bioavailability, and micro- and macrovascular disease. ${ }^{39-41}$ On the other hand, insulin resistance facilitates the development of hypertension by multiple mechanisms. ${ }^{39,40,42}$ The most direct link is the well-established stimulation of the sympathetic drive by hyperinsulinemia. ${ }^{43,44}$ Besides, chronic inflammation, oxidative stress, and activation of the renin-angiotensin-aldosterone system further facilitate initiation and maintenance of hypertension. ${ }^{39,40,45}$ On the other hand, physical activity acutely improves insulin sensitivity, and regular exercise induces adaptations counteracting most of the pathophysiological mechanisms involved in the development of insulin resistance and to some extent, hypertension. ${ }^{46}$ In agreement, after accounting for physical activity, the observed impairment in insulin sensitivity in the hypertensives was much attenuated in the present investigation.

\section{4 | Limitations}

The current observations are based on a cross-sectional study. Nevertheless, in contrast with previous studies, we assessed body composition, cardiorespiratory fitness, and physical activity with state-of-the-art methods. Although no significant differences were observed in RMR between treated and untreated hypertensive patients, we cannot rule out a reduction of RMR in the hypertensive patients with a more intense or different pharmacological treatment. For example, beta-blockers could reduce RMR by lowering resting heart rate and counteracting the effects of sympathetic overactivation. ${ }^{1,47}$

In summary, the present study indicates that hypertension is associated with increased RMR and reduced insulin sensitivity. Our research demonstrates that the increased resting metabolism is explained primarily by an increased myocardial oxygen consumption due to the elevated resting double product, combined with differences in body composition between hypertensive and normotensive. The reduced insulin sensitivity in hypertensive patients was mostly accounted for 
by differences in body composition, physical activity, and cardiorespiratory fitness.

\section{5 | PERSPECTIVE}

Future studies should address whether changes in resting double product elicited by exercise training or pharmacological treatments modify the resting metabolic rate and energy balance. Since the reduced insulin sensitivity observed in hypertensive patients is accounted for by cardiorespiratory fitness and physical activity, both variables could be easily improved by exercise prescription. Since the results here reported were obtained in young and middle-aged Caucasians, future studies should also examine whether RMR is also increased in hypertensives of other ethnicities and elderly patients.

\section{ACKNOWLEDGEMENTS}

The technical assistance of Jose Navarro de Tuero is much appreciated.

\section{CONFLICT OF INTEREST}

The authors declare that they have no conflict of interest.

\section{DATA AVAILABILITY STATEMENT}

Due to confidentiality agreements, supporting data can only be made available to bona fide researchers subject to a nondisclosure agreement upon direct request to the corresponding author.

\section{ORCID}

Pablo B. Pedrianes-Martin (10) https://orcid. org/0000-0001-9173-500X

Mario Perez-Valera (10) https://orcid. org/0000-0002-8332-729X

David Morales-Alamo (1) https://orcid.

org/0000-0001-8463-397X

Marcos Martin-Rincon (1) https://orcid.

org/0000-0002-3685-2331

Ismael Perez-Suarez (10 https://orcid.

org/0000-0002-7969-0061

Jose A. Serrano-Sanchez (i) https://orcid. org/0000-0002-4868-8751

Victor Galvan-Alvarez (10) https://orcid. org/0000-0002-1272-3471

David Curtelin (D) https://orcid.org/0000-0001-6462-382X

Jose A. L. Calbet (D) https://orcid.org/0000-0002-9215-6234

\section{REFERENCES}

1. Kunz I, Schorr U, Klaus S, Sharma AM. Resting metabolic rate and substrate use in obesity hypertension. Hypertension. 2000;36(1):26-32.
2. Creber C, Cooper RS, Plange-Rhule J, et al. Independent association of resting energy expenditure with blood pressure: confirmation in populations of the African diaspora. BMC Cardiovasc Disord. 2018;18(4):1-10.

3. Grassi G, Dell'Oro R, Facchini A, Quarti Trevano F, Bolla GB, Mancia G. Effect of central and peripheral body fat distribution on sympathetic and baroreflex function in obese normotensives. $J$ Hypertens. 2004;22(12):2363-2369.

4. Claflin KE, Sandgren JA, Lambertz AM, et al. Angiotensin AT1A receptors on leptin receptor-expressing cells control resting metabolism. J Clin Invest. 2017;127(4):1414-1424.

5. Johnstone AM, Murison SD, Duncan JS, Rance KA, Speakman JR. Factors influencing variation in basal metabolic rate include fat-free mass, fat mass, age, and circulating thyroxine but not sex, circulating leptin, or triiodothyronine. Am J Clin Nutr. 2005;82(5):941-948.

6. Muller MJ, Bosy-Westphal A, Kutzner D, Heller M. Metabolically active components of fat-free mass and resting energy expenditure in humans: recent lessons from imaging technologies. Obes Rev. 2002;3(2):113-122.

7. da Silva AA, do Carmo JM, Li X, Wang Z, Mouton AJ, Hall JE. Role of hyperinsulinemia and insulin resistance in hypertension: metabolic syndrome revisited. Can J Cardiol. 2020;36(5):671-682.

8. Roberts CK, Hevener AL, Barnard RJ. Metabolic syndrome and insulin resistance: underlying causes and modification by exercise training. Compr Physiol. 2013;3(1):1-58.

9. Cancello R, Soranna D, Brunani A, et al. Analysis of predictive equations for estimating resting energy expenditure in a large cohort of morbidly obese patients. Front Endocrinol. 2018;9(367):1-8.

10. Rolfe DF, Brown GC. Cellular energy utilization and molecular origin of standard metabolic rate in mammals. Physiol Rev. 1997;77(3):731-758.

11. Hoeft A, Sonntag H, Stephan H, Kettler D. Validation of myocardial oxygen demand indices in patients awake and during anesthesia. Anesthesiology. 1991;75(1):49-56.

12. Martin-Rincon M, Perez-Valera M, Morales-Alamo D, et al. Resting energy expenditure and body composition in overweight men and women living in a temperate climate. J Clin Med. 2020;9(203):1-20.

13. Calbet JAL, Ponce-González JG, Calle-Herrero JDL, et al. Exercise preserves lean mass and performance during severe energy deficit: the role of exercise volume and dietary protein content. Front Physiol. 2017;8(483):1-13.

14. Pickering TG, Hall JE, Appel LJ, et al. Recommendations for blood pressure measurement in humans and experimental animals: part 1: blood pressure measurement in humans: a statement for professionals from the Subcommittee of Professional and Public Education of the American Heart Association Council on High Blood Pressure Research. Hypertension. 2005;45(1):142-161.

15. Marra M, Cioffi I, Sammarco R, et al. Prediction and evaluation of resting energy expenditure in a large group of obese outpatients. Int J Obes. 2017;41(5):697-705.

16. Perez-Suarez I, Martin-Rincon M, Gonzalez-Henriquez JJ, et al. Accuracy and precision of the COSMED K5 portable analyser. Front Physiol. 2018;9(1764):1-12.

17. Peronnet F, Massicotte D. Table of nonprotein respiratory quotient: an update. Can J Sport Sci. 1991;16(1):23-29.

18. Poole DC, Wilkerson DP, Jones AM. Validity of criteria for establishing maximal $\mathrm{O}_{2}$ uptake during ramp exercise tests. Eur J Appl Physiol. 2008;102(4):403-410. 
19. Martin-Rincon M, Gonzalez-Henriquez JJ, Losa-Reyna J, et al. Impact of data averaging strategies on $\mathrm{VO}_{2 \max }$ assessment: mathematical modeling and reliability. Scand J Med Sci Sports. 2019;29(10):1473-1488.

20. Menendez E, Delgado E, Fernandez-Vega F, et al. Prevalence, diagnosis, treatment, and control of hypertension in spain. Results of the Di@ bet.es Study. Rev Esp Cardiol. 2016;69(6):572-578.

21. Pourhassan M, Bosy-Westphal A, Schautz B, Braun W, Gluer CC, Muller MJ. Impact of body composition during weight change on resting energy expenditure and homeostasis model assessment index in overweight nonsmoking adults. Am J Clin Nutr. 2014;99(4):779-791.

22. Grassi G, Mark A, Esler M. The sympathetic nervous system alterations in human hypertension. Circ Res. 2015;116(6):976-990.

23. Fu Q. Sex differences in sympathetic activity in obesity and its related hypertension. Ann N Y Acad Sci. 2019;1454(1):31-41.

24. Kim D-I, Yang HI, Park J-H, et al. The association between resting heart rate and type 2 diabetes and hypertension in Korean adults. Heart. 2016;102(21):1757-1762.

25. Adachi H, Enomoto M, Fukami A, et al. Plasma renin activity and resting heart rate in a population of community-dwelling Japanese: the Tanushimaru Study. Am J Hypertens. 2015;28(7):894-899.

26. Wang ZiMian, Ying Z, Bosy-Westphal A, et al. Specific metabolic rates of major organs and tissues across adulthood: evaluation by mechanistic model of resting energy expenditure. Am J Clin Nutr. 2010;92(6):1369-1377.

27. Oral EA, Simha V, Ruiz E, et al. Leptin-replacement therapy for lipodystrophy. N Engl J Med. 2002;346(8):570-578.

28. Kaiyala KJ, Morton GJ, Leroux BG, Ogimoto K, Wisse B, Schwartz MW. Identification of body fat mass as a major determinant of metabolic rate in mice. Diabetes. 2010;59(7):1657-1666.

29. Karsenty G. Convergence between bone and energy homeostases: leptin regulation of bone mass. Cell Metab. 2006;4(5):341-348.

30. Klieverik LP, Coomans CP, Endert E, et al. Thyroid hormone effects on whole-body energy homeostasis and tissue-specific fatty acid uptake in vivo. Endocrinology. 2009;150(12):5639-5648.

31. Vaz M, Jennings G, Turner A, Cox H, Lambert G, Esler M. Regional sympathetic nervous activity and oxygen consumption in obese normotensive human subjects. Circulation. 1997;96(10):3423-3429.

32. Reinehr T. Obesity and thyroid function. Mol Cell Endocrinol. 2010;316(2):165-171.

33. Sjøberg KA, Frøsig C, Kjøbsted R, et al. Exercise increases human skeletal muscle insulin sensitivity via coordinated increases in microvascular perfusion and molecular signaling. Diabetes. 2017;66(6):1501-1510.

34. Richter EA, Hargreaves M. Exercise, GLUT4, and skeletal muscle glucose uptake. Physiol Rev. 2013;93(3):993-1017.

35. Barrett EJ, Eggleston EM, Inyard AC, et al. The vascular actions of insulin control its delivery to muscle and regulate the rate-limiting step in skeletal muscle insulin action. Diabetologia. 2009;52(5):752-764.

36. Sena CM, Pereira AM, Seica R. Endothelial dysfunction - a major mediator of diabetic vascular disease. Biochim Biophys Acta. 2013;1832(12):2216-2231.

37. Julius S, Gudbrandsson T, Jamerson K, Tariq Shahab S, Andersson O. The hemodynamic link between insulin resistance and hypertension. J Hypertens. 1991;9(11):983-986.

38. Jamerson KA, Julius S, Gudbrandsson T, Andersson O, Brant DO. Reflex sympathetic activation induces acute insulin resistance in the human forearm. Hypertension. 1993;21(5):618-623.

39. Koliaki C, Liatis S, Kokkinos A. Obesity and cardiovascular disease: revisiting an old relationship. Metabolism. 2019;92:98-107.

40. Petrie JR, Guzik TJ, Touyz RM. Diabetes, hypertension, and cardiovascular disease: clinical insights and vascular mechanisms. Can J Cardiol. 2018;34(5):575-584.

41. Mahfoud F, Schlaich M, Kindermann I, et al. Effect of renal sympathetic denervation on glucose metabolism in patients with resistant hypertension: a pilot study. Circulation. 2011;123(18):1940-1946.

42. Anderson EA, Hoffman RP, Balon TW, Sinkey CA, Mark AL. Hyperinsulinemia produces both sympathetic neural activation and vasodilation in normal humans. J Clin Invest. 1991;87(6):2246-2252.

43. Denker PS, Pollock VE. Fasting serum insulin levels in essential hypertension. Arch Intern Med. 1992;152(8):1649-1651.

44. Ferrannini E, Cushman WC. Diabetes and hypertension: the bad companions. Lancet. 2012;380(9841):601-610.

45. Khodabandehloo H, Gorgani-Firuzjaee S, Panahi G, Meshkani R. Molecular and cellular mechanisms linking inflammation to insulin resistance and beta-cell dysfunction. Transl Res. 2016;167(1):228-256

46. Lin X, Zhang XI, Guo J, et al. Effects of exercise training on cardiorespiratory fitness and biomarkers of cardiometabolic health: a systematic review and meta-analysis of randomized controlled trials. J Am Heart Assoc. 2015;4(7):1-28.

47. Buemann B, Astrup A, Madsen J, Christensen NJ. A 24-h energy expenditure study on reduced-obese and nonobese women: effect of beta-blockade. Am J Clin Nutr. 1992;56(4):662-670.

How to cite this article: Pedrianes-Martin PB, Perez-

Valera M, Morales-Alamo D, et al. Resting metabolic rate is increased in hypertensive patients with overweight or obesity: Potential mechanisms. Scand J Med Sci Sports. 2021;31:1461-1470. https://doi.org/10.1111/sms.13955 